\title{
Dosage simultané des phosphates et des chlorures dans les ultrafiltrats de lait par titrage argentimétrique à $\mathrm{pH}$ constant
}

\author{
par \\ J.-F. LE MEUR, H. LE NOURS et C. BOURGEOIS \\ avec la collaboration technique de Marie-Hélène GLEMAREC \\ et J. C. COCHET
}

\section{R és u m é}

La détermination simultanée des ions phosphate et chlorure dans des ultrafiltrats de lait peut être obtenue par titrage argentimétrique à $\mathrm{pH}$ régulé. Cette détermination s'effectue par l'intermédiaire du tracé des courbes donnant le volume de soude nécessaire au maintien du $\mathrm{pH}$ constant, en fonction de l'ajout d'argent. Le choix du $\mathrm{pH}(\mathrm{pH}=8)$, l'utilisation préalable d'une déminéralisation sur résine échangeuse de cations permettent d'éliminer les interférences. Les résultats obtenus par cette technique et ceux de la méthode spectrométrique (phosphate) et potentiométrique (chlorures) présentent une bonne cohérence.

Mots clés : Ultrafiltrat de lait - Phosphate - Chlorure - Titrage à $\mathrm{pH}$ régulé Argentimétrie.

\section{S u m m a r y}

USE OF A PH STAT FOR THE SIMULTANEOUS PHOSPHATE AND CHLORIDE DETERMINATION IN MILK ULTRAFILTRATES

The quantity of phosphate and chloride present in milk ultrafiltrates can be measured by means of a silver titration analysis with à $\mathrm{pH}$ stat. This determination can be read on a curve giving the volume of sodium hydroxide necessary to keep the $\mathrm{pH}$ constant in proportion of the silver amount. By choosing a $p H 8$ and demineralizing before the ultrafiltrates on cation exchangers, we can

Laboratoire de Recherche agro-alimentaire, Institut Universitaire de technologie, rue de l'Université, B.P. 319 - 29191 Quimper cedex. 
get rid of interferences. The results obtained with this technique and with the spectrometric (phosphate) and potentiometric (chloride) techniques show a good coherence.

Key words: Milk ultrafiltrate - Phosphate - Chloride - pH stat - Silver titration.

\section{INTRODUCTION}

L'influence du calcium, des ions citrate, et phosphate sur la stabilité des micelles de caséine a été mise en évidence par de nombreux auteurs entre autres (White, Davies 1958 ; Visser et coll. 1979; Sleigh et coll. 1979). En effet les équilibres régissant les espèces minérales existant dans la phase soluble sont tels, que de faibles variations de l'un des paramètres peuvent les modifier fortement. Il est donc souhaitable de disposer de méthodes rapides d'analyse des espèces dissoutes afin de mieux maîtriser les processus technologiques de transformation du lait tels que caséinerie et fromagerie.

Nous avons précédemment mis au point une méthode de dosage des ions citrate. Cette technique basée sur un titrage complexométrique par le cuivre à $\mathrm{pH}$ contrôlé (Le Nours, Le Meur, Bourgeois 1983) peut être appliquée à de nombreux ions minéraux et organiques (Le Meur, Le Nours, 1984).

Dans le présent travail, nous indiquons les possibilités de cette méthode pour le dosage simultané des ions phosphate et chlorure et nous comparons nos résultats avec ceux des méthodes spectrométrique (phosphomolybdate) et potentiométrique (électrode d'argent) pour des ultrafiltrats de lait.

\section{PRINCIPE DE LA METHODE}

Le phosphate forme avec de nombreux métaux des précipités faisant intervenir l'ion $\mathrm{PO}_{4}{ }^{3-}$. Cette réaction peut entraîner une libération de protons susceptible d'être mesurée. La présence des ions citrate dans les ultrafiltrats de lait, qui peuvent jouer le rôle d'agent complexant, et une mauvaise définition de la stoechiométrie de certains précipités, nous ont conduits à exclure des métaux tels que $\mathrm{Cu}^{2+}$, $\mathrm{Fe}^{3+}, \mathrm{Mg}^{2+}, \mathrm{Pb}^{2+}$ et à choisir l'ion $\mathrm{Ag}^{+}$comme réactif titrant.

Les réactions de précipitation qui se produisent sont :

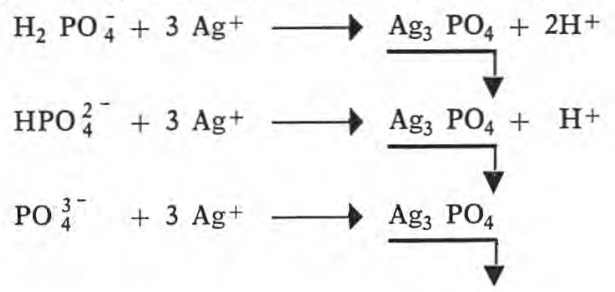


Dans les ultrafiltrats de lait la présence des ions chlorure entraîne une précipitation de chlorure d'argent. Les produits de solubilité des deux espèces sont : $\mathrm{K}_{\mathrm{s}}(\mathrm{AgCl})=10^{-10}, \mathrm{~K}_{\mathrm{s}}\left(\mathrm{Ag}_{3} \mathrm{PO}_{4}\right)=10^{-21}$ (Sillen, Martell, 1964). Une modélisation du phénomène (Le Meur, Le Nours, 1984) met en évidence que la précipitation de $\mathrm{AgCl}$ intervient d'abord, puis on observe dans un deuxième temps celle de $\mathrm{Ag}_{3} \mathrm{PO}_{4}$. Le modèle mathématique élaboré donne le nombre de moles de $\mathrm{H}^{+}$ libérées en fonction de la quantité $\mathrm{d}^{\prime} \mathrm{Ag}^{+}$fourni.

Le $\mathrm{pH}$ est maintenu constant à l'aide d'une chaîne de $\mathrm{pH}$ stat et donc la libération de protons entraîne une consommation d'ions $\mathrm{OH}^{-}$délivrés par la burette automatique sous forme de soude.

Le calcul est basé sur les bilans matière suivants :

$$
\begin{aligned}
\mathrm{V}_{\mathrm{A}} \times\left[\mathrm{PO}_{4}^{3-}\right] \text { tot } & =\left(\left[\mathrm{PO}_{4}^{3-}\right]+\left[\mathrm{HPO}_{4}^{2-}\right]+\left[\mathrm{H}_{2} \mathrm{PO}_{4}^{-}\right]+\left[\mathrm{H}_{3} \mathrm{PO}_{4}\right]\right) \times \mathrm{V}_{\mathrm{T}}+\mathrm{n}_{1} \mathrm{Ag}_{3} \mathrm{PO}_{4} \\
& =\left[\mathrm{PO}_{4}^{3-}\right] \times\left(1+\frac{\left[\mathrm{H}^{+}\right]}{-\mathrm{K}_{\mathrm{A} 1}}+\frac{\left[\mathrm{H}^{+}\right]^{2}}{\mathrm{Al}^{\times \mathrm{K}_{\mathrm{A} 2}}}+\frac{\left[\mathrm{H}^{+}\right]^{3}}{\mathrm{~K}_{\mathrm{Al}} \mathrm{K}_{\mathrm{A} 2} \mathrm{~K}_{\mathrm{A} 3}}\right) \times \mathrm{V}_{\mathrm{T}}+\mathrm{n}_{\mathrm{I}}^{+} \mathrm{Ag}_{3} \mathrm{PO}_{4} \\
\mathrm{~V}_{\mathrm{M}} \times\left[\mathrm{Ag}^{+}\right] \text {tot } & =\left[\mathrm{Ag}^{+}\right] \times \mathrm{V}_{\mathrm{T}}+3 \mathrm{n}_{2} \mathrm{Ag}_{3} \mathrm{PO}_{4}+\mathrm{n}_{2} \mathrm{Ag} \mathrm{Cl} \\
\mathrm{V}_{\mathrm{A}} \times\left[\mathrm{CI}^{-}\right]_{\text {tot }} & =\left[\mathrm{CI}^{-}\right] \times \mathrm{V}_{\mathrm{T}}+\mathrm{n}_{2} \mathrm{Ag} \mathrm{CI}
\end{aligned}
$$

Avec $\mathrm{V}=$ Volume du mélange contenant l'acide.

$$
\begin{aligned}
\mathrm{V}_{\mathrm{M}}^{\mathrm{A}} & =\text { Volume d'argent ajouté. } \\
\mathrm{V}_{\mathrm{T}} & =\text { Volume total. } \\
& =\text { Volume d'acide } \mathrm{V}_{\mathrm{A}}+\text { volume métal } \mathrm{V}_{\mathrm{M}}+\text { volume } \\
& \text { d'eau } \mathrm{V}_{\mathrm{E}} \text {. }
\end{aligned}
$$

$\mathrm{K}_{\mathrm{A} 1}, \mathrm{~K}_{\mathrm{A2}}, \mathrm{K}_{\mathrm{A3}}=$ Constantes d'acidité de l'acide phosphorique $\left(\mathrm{pK}_{\mathrm{A}}=11,7 ; 6,8 ; 2\right)$.

$\mathrm{n}_{1} \mathrm{Ag}_{3} \mathrm{PO}_{4}$, et $\mathrm{n}_{2} \mathrm{Ag} \mathrm{Cl}$ correspondent au nombre de moles de précipités.

et les produits de solubilité,

$$
\mathrm{K}_{\mathrm{S} 1}=\left[\mathrm{Ag}^{+}\right]^{3} \times\left[\mathrm{PO}_{4}^{3-}\right] \quad \mathrm{K}_{\mathrm{S} 2}=\left[\mathrm{Ag}^{+}\right] \times\left[\mathrm{CI}^{-}\right]
$$

Pour des concentrations d'argent et de phosphate telles que le produit de solubilité n'est pas atteint, on annulera $\mathrm{n}_{1}$ dans les calculs.

Si on prend comme variable $V_{M}$, une résolution de ce système d'équation permet d'obtenir le nombre de moles d'espèces précipitées et par suite le volume de soude nécessaire pour maintenir constant le $\mathrm{pH}$. On observe que cette courbe présente trois segments de droite : 


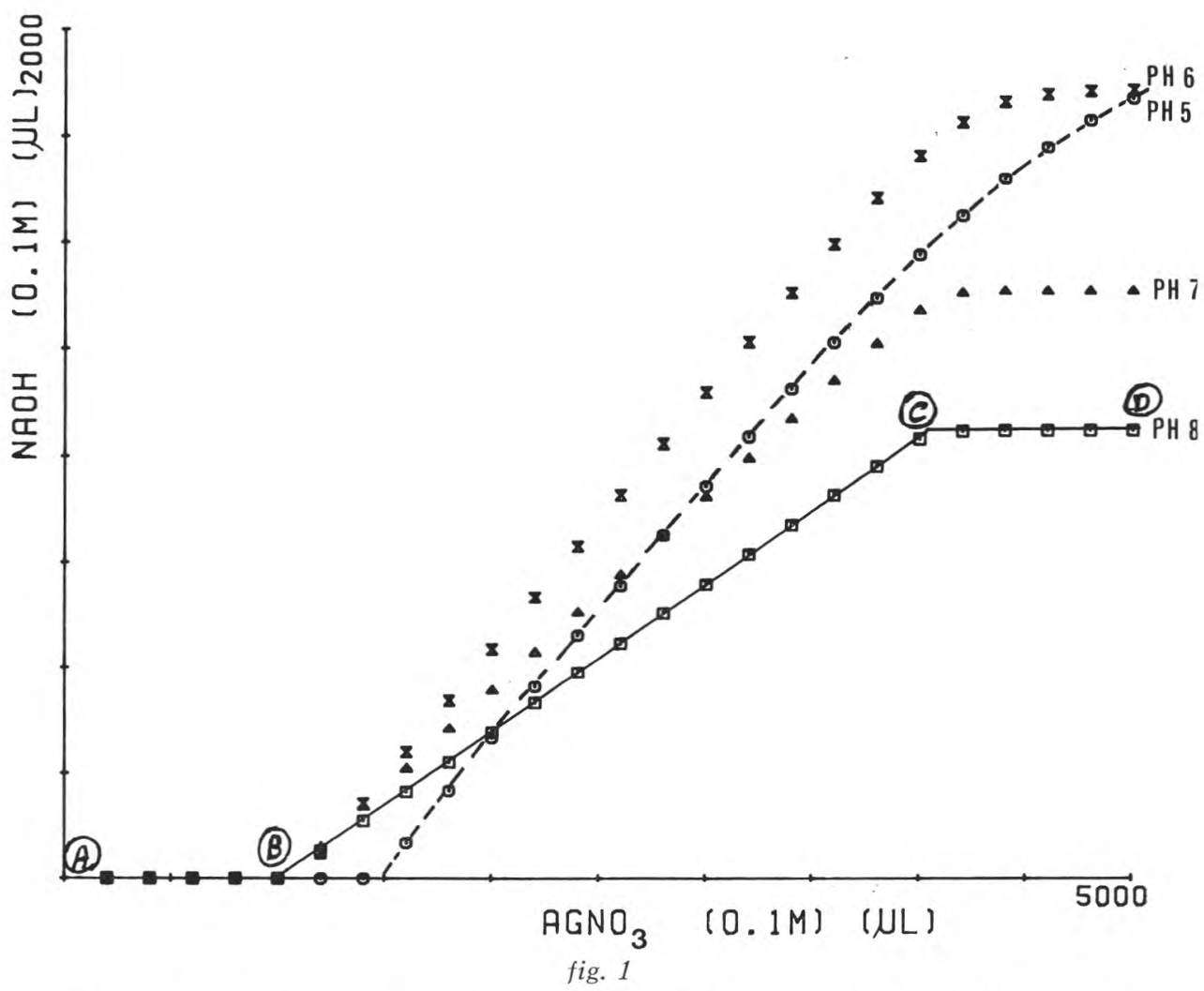

Evolution avec le $\mathrm{pH}$ des courbes de titrage d'un mélange phosphate-chlorure (10 ml phosphate $10^{-2} \mathrm{M}+10 \mathrm{ml}$ chlorure $10-2 \mathrm{M}$ par du nitrate d'argent $0,1 \mathrm{M}$ ). Courbes obtenues par le calcul.

Variation with $\mathrm{pH}$ of the titration curves of a phosphate-chloride mixture $(10 \mathrm{ml}$ $10-2 \mathrm{M}$ phosphate $+10 \mathrm{ml} 10-2 \mathrm{M}$ chloride with 0,1M silver nitrate). Theoretical curves.

$\mathrm{AB}, \mathrm{BC}, \mathrm{CD}$ (fig. 1). Les segments $\mathrm{AB}$ et $\mathrm{BC}$ correspondent respectivement à la précipitation $\mathrm{d}^{\prime} \mathrm{AgCl}$ sans libération de protons et à celle d'Ag $\mathrm{PO}_{4}$ avec libération de protons. Le segment CD correspond à l'ajout d'un excès de nitrate d'argent. Les points $\mathrm{B}$ et $\mathrm{C}$ peuvent donc être utilisés pour le calcul des concentrations en chlorure et phosphate.

\section{MATERIELS ET TECHNIQUES}

\section{La chaîne de mesure}

Elle comprend un pH mètre E 632, une unité de contrôle E 614 et une burette automatique $\mathrm{E} 655$, avec un corps de $5 \mathrm{ml}$. Le réactif est délivré par une conduite munie d'une pointe anti-diffusion, ce 
qui permet une précision de l'ordre du microlitre. L'ensemble est commercialisé par la société Metrohm.

Les mesures sont effectuées à température constante $\left(25^{\circ} \mathrm{C}\right)$ dans une cellule de $100 \mathrm{ml}$ munie d'une enceinte à circulation reliée à un thermostat Haake.

Le $\mathrm{pH}$ mètre est standardisé à 0,01 unité de $\mathrm{pH}$ par deux tampons $(7,12-4,64)$.

\section{Spectrométrie des phosphates et dosage des chlorures}

Les mesures de densité optique sont effectuées à $825 \mathrm{~nm}$ au moyen d'un spectrophotomètre Jean et Constant et reposent sur la détermination du complexe phosphomolybdate réduit (Duval, 1963 ; Brzenk, Krett, 1976).

Les chlorures sont déterminés par potentiomètrie avec électrode d'argent en présence d'H $\mathrm{NO}_{3} \mathrm{~N}$.

\section{Préparation des échantillons}

Les ultrafiltrats sont obtenus avec un appareillage DDS muni de 18 membranes du type GR61P en polysulfone. Les échantillons sont des laits reconstitués par dissolution de lait en poudre dans de l'eau déminéralisée.

\section{Déminéralisation}

Elle est effectuée par passage sur résine Amberlite IR 120 sous forme $\mathrm{H}^{+}$(Christienson, Jennes, Coulter 1954).

Dans un bécher de $100 \mathrm{ml}$, on introduit environ $10 \mathrm{ml}$ de résine. On ajoute $50 \mathrm{ml}$ d'acide nitrique $\mathrm{N}$ pour mettre la résine sous forme acide. Après rinçage de la résine par de l'eau distillée, on introduit la prise d'essai (10 à $20 \mathrm{ml})$. Ce volume est choisi en fonction de la teneur moyenne en phosphate d'un ultrafiltrat, afin d'avoir environ $10^{-4}$ moles de phosphate dans la cellule de mesure. Après une agitation de $10 \mathrm{~min}$, on filtre l'échantillon sur laine de verre et on rince deux fois la résine par $15 \mathrm{ml}$ d'eau distillée; les eaux de rinçage sont jointes à l'échantillon.

\section{Méthodes de dosage}

L'échantillon déminéralisé, les eaux de rinçage et $1 \mathrm{ml}$ d'acide borique $10^{-1} \mathrm{M}$ sont introduits dans la cellule de mesure. Le pH est amené à 8 par addition de soude $0,1 \mathrm{M}$. Après remise à zéro de la burette, on effectue des ajouts de nitrate d'argent $0,1 \mathrm{M}$ (volume : $500 \mu \mathrm{l}$ ) et on note pour chaque ajout le volume de soude nécessaire au retour à $8 \mathrm{du} \mathrm{pH}$. 


\section{Traitement des données}

Nous avons mis au point pour cette étude un programme sur micro-ordinateur Apple II. Celui-ci après saisie des données, inscrit les points expérimentaux, fait la régression linéaire sur les deux segments de droite de la courbe de titrage après rejet automatique des points situés au voisinage du point équivalent et détermine la concentration en phosphate et chlorure de l'échantillon.

\section{RESULTATS ET DISCUSSION}

\section{Choix des conditions opératoires}

Le modèle précédemment proposé nous a permis une recherche des conditions optimales de travail et ceci a été confirmé expérimentalement : sur la figure 1 nous constatons qu'un abaissement du $\mathrm{pH}$ entraîne une consommation plus importante d'ions $\mathrm{OH}^{-}$due à un plus fort pourcentage des formes protonées, puis on observe l'effet inverse dû à la précipitation incomplète.

De plus pour des $\mathrm{pH}$ supérieurs à 8,5 la précipitation de $\mathrm{Ag} \mathrm{OH}$ peut perturber le dosage en consommant des ions $\mathrm{OH}^{-}$.

Compte tenu de ces différents paramètres, nous avons choisi le pH 8 comme pH optimal de mesure.

D'autre part, en fin de précipitation la disparition des ions phosphate diminue l'effet tampon de la solution et rend difficile la stabilisation du $\mathrm{pH}$, ceci nous a donc conduits à ajouter de l'acide borique qui présente un bon pouvoir tampon à $\mathrm{pH}$ 8. Nous avons vérifié que l'ajout de cet acide ne modifiait pas la précipitation du phosphate d'argent.

\section{Interférences}

Nous avons étudié l'influence de différentes espèces présentes dans les ultrafiltrats et susceptibles de réagir avec les ions $\mathrm{Ag}^{+}$, ou de complexer les ions phosphate; pour ce faire, on effectue dans des solutions synthétiques des ajouts de ces substances aux concentrations voisines de celles habituellement rencontrées dans les ultrafiltrats.

\subsection{Calcium et magnésium}

Ces éléments forment avec les ions phosphate des complexes et peuvent modifier les courbes de titrage (fig. 2). Il est donc nécessaire de les éliminer au préalable par passage sur résine échangeuse de cations.

\subsection{Citrate}

Nous avons vérifié en effectuant des ajouts de citrate jusqu'à 1,5. $10^{-2}$ mole/1 dans une solution synthétique contenant des chlo- 


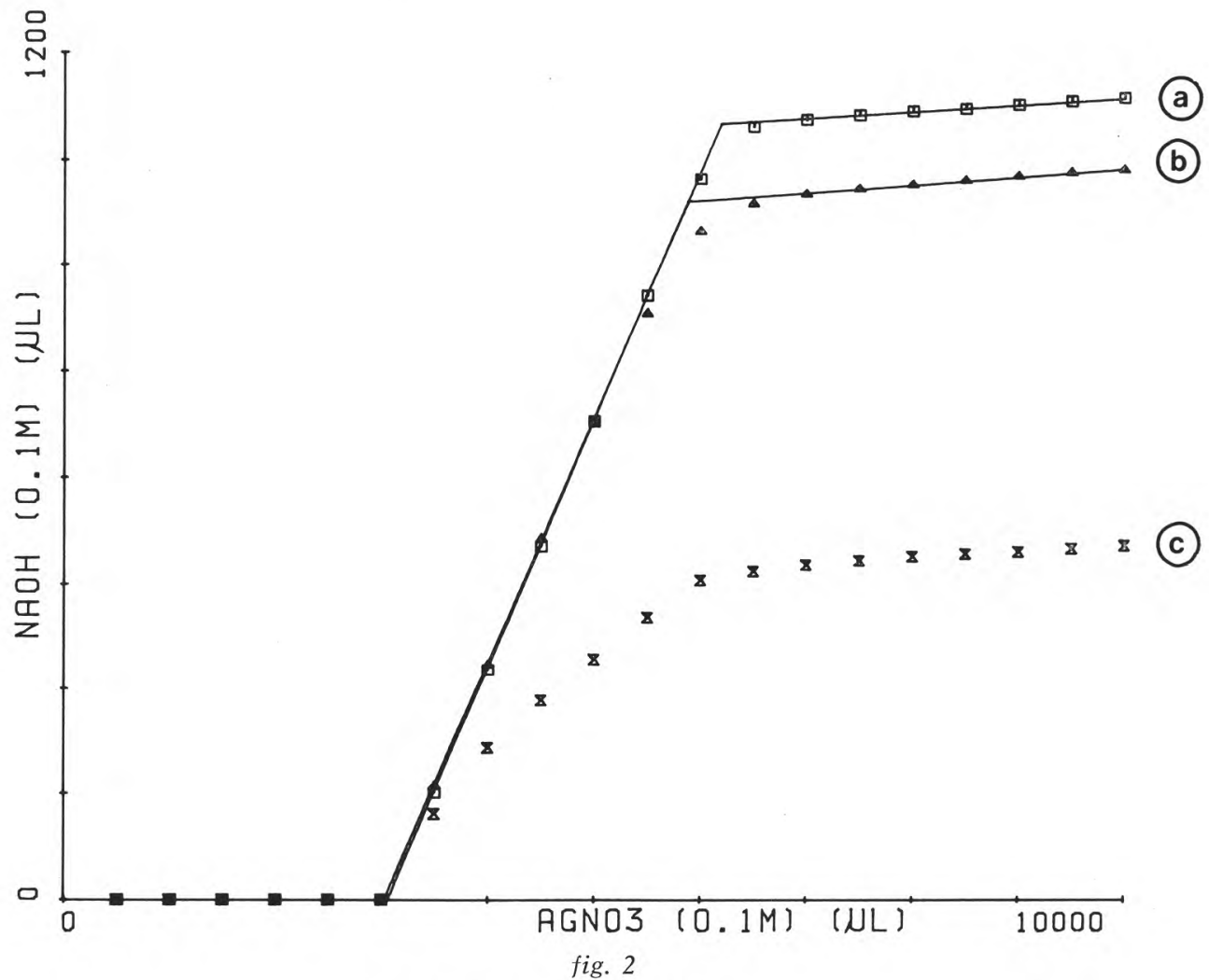

Interférence des ions calcium à $\mathrm{pH} 8$

a) $3 \mathrm{ml}$ chlorure $10^{-1} \mathrm{M}+10 \mathrm{ml}$ phosphate $10^{-2} \mathrm{M}+10 \mathrm{ml}$ citrate $10^{-2} \mathrm{M}+1 \mathrm{ml}$ acide borique $10^{-1} \mathrm{M}+20 \mathrm{ml} \mathrm{KNO}_{3}{ }^{10-1} \mathrm{M}$.

b) id. a) $+1 \mathrm{ml}$ calcium $10-1 \mathrm{M}$

c) id. b) $+1,5 \mathrm{ml}$ calcium $10-1 \mathrm{M}$.

Interaction of calcium ion at $\mathrm{pH} 8$

a) $3 \mathrm{ml} 10-1 \mathrm{M}$ chloride $+10 \mathrm{ml} 10-2 \mathrm{M}$ phosphate $+10 \mathrm{ml} 10-2 \mathrm{M}$ citrate $+1 \mathrm{ml}$ $10^{-1} \mathrm{M}$ boric acid $+20 \mathrm{ml} 10^{-1} \mathrm{M} \mathrm{KNO}_{3}$.

b) id. a) $+1 \mathrm{ml}$ 10-1M calcium.

c) id. b) $+1,5 \mathrm{ml} 10^{-1} \mathrm{M}$ calcium.

rures (3. $10^{-2}$ mole/l) et des phosphates $\left(10^{-2}\right.$ mole/l) que cette addition n'avait pas d'influence significative sur les résultats.

On observe cependant une légère modification de la courbe après le deuxième point équivalent (fig. 3). Ceci peut être dû à la formation d'un complexe entre l'argent et la forme totalement déprotonée de l'ion citrate. 


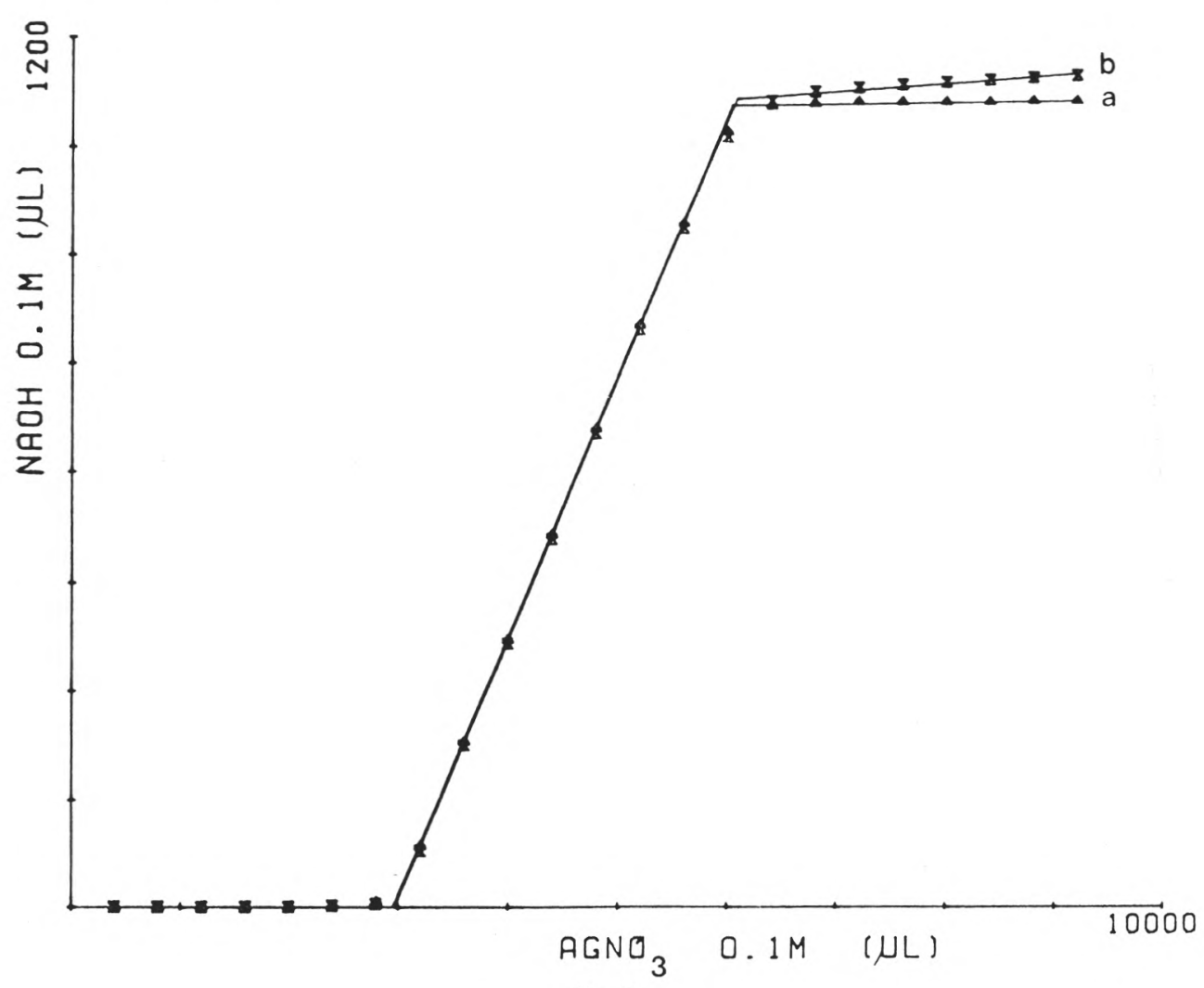

fig. 3

Interférence des ions citrate à $\mathrm{pH} 8$

a) $3 \mathrm{ml}$ chlorure $10-1 \mathrm{M}+10 \mathrm{ml}$ phosphate $10^{-2} \mathrm{M}+1 \mathrm{ml}$ acide borique $10^{-1} \mathrm{M}+$ $20 \mathrm{ml} \mathrm{KNO}_{3} 10^{-1} \mathrm{M}$.

b) id. a) $+0,8 \mathrm{ml}$ citrate $10-1 \mathrm{M}$.

Interaction of citrate ion at $\mathrm{pH} 8$

a) $3 \mathrm{ml} \mathrm{10-1} \mathrm{M}$ chloride $+10 \mathrm{ml} 10^{-2} \mathrm{M}$ phosphate $+1 \mathrm{ml} 10^{-1} \mathrm{M}$ boric acid + $20 \mathrm{ml} 10^{-1} \mathrm{M} \mathrm{KNO}$.

b) id. a) $+0,8 \mathrm{ml} 10-1 \mathrm{M}$ citrate.

\subsection{Substances comportant UN GROUPE aminÉ}

Celles-ci sont susceptibles de réagir avec les ions $\mathrm{Ag}^{+}$, mais nous avons vérifié que l'étape de déminéralisation préalable en milieu acide permettait d'éliminer simultanément calcium, magnésium et substances aminées. 


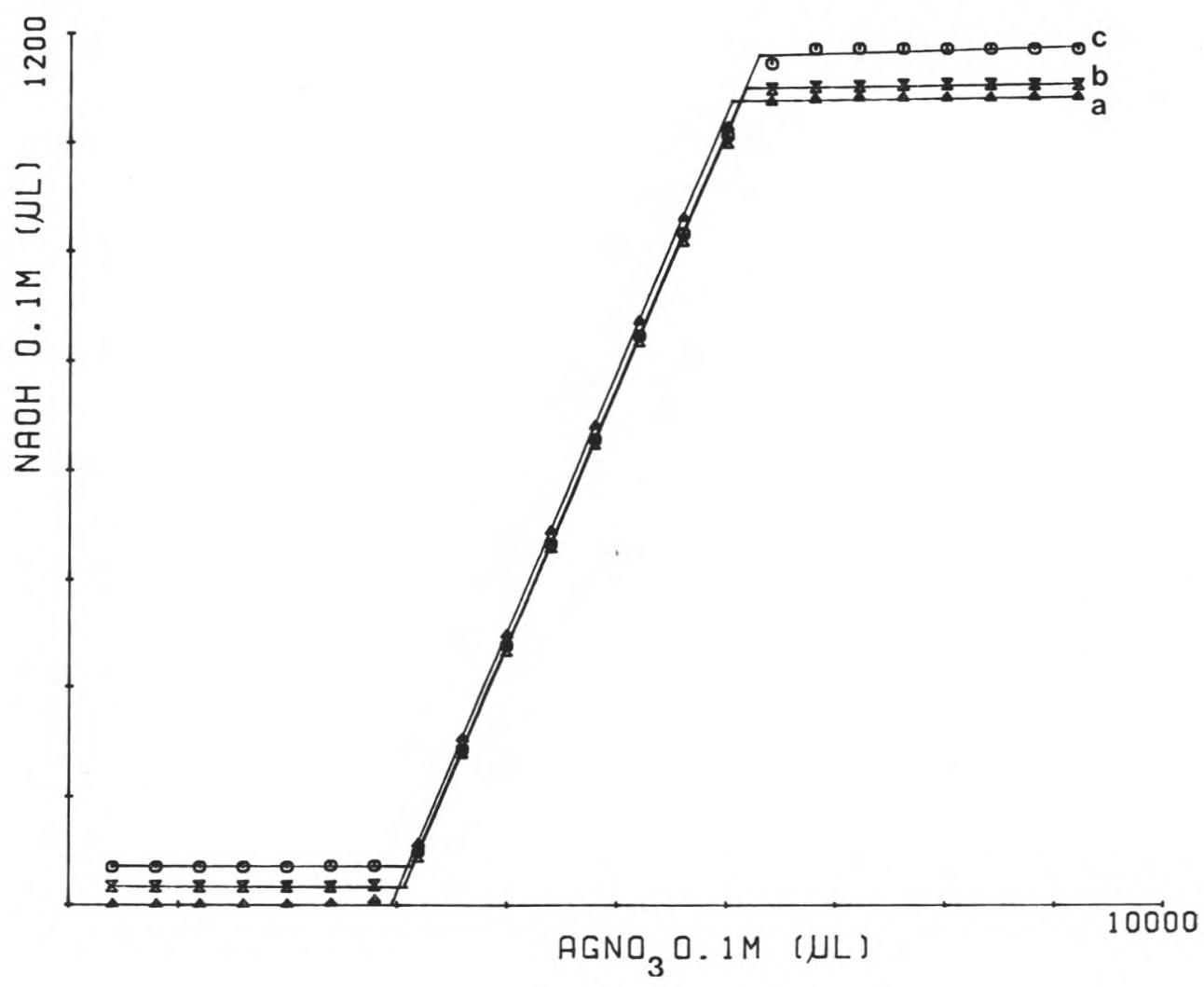

fig. 4

Influence des sulfures sur le titrage à $\mathrm{pH} 8$ d'un mélange phosphate chlorure (3 ml chlorure $10^{-1} \mathrm{M}+10 \mathrm{ml}$ phosphate ${ }^{10-2} \mathrm{M}+1 \mathrm{ml}$ acide borique ${ }^{10-1} \mathrm{M}+$ $\left.20 \mathrm{ml} \mathrm{KNO}_{3} \quad 10-1 \mathrm{M}\right)$.

a) Sans sulfure.

b) $+0,3 \mathrm{ml}$ sulfure de sodium $10-2 \mathrm{M}$.

c) $+0,6 \mathrm{ml}$ sulfure de sodium $10-2 \mathrm{M}$.

Interaction of sulphur ions at $\mathrm{pH} 8$.

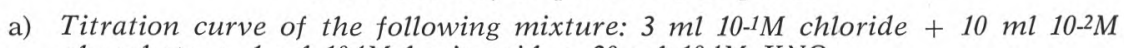
phosphate $+1 \mathrm{ml} \mathrm{10}^{-1} \mathrm{M}$ boric acid $+20 \mathrm{ml}^{1} \mathrm{l}^{-1} \mathrm{M} \mathrm{KNO} \mathrm{K}_{3}$.

b) id. a) + 0,3 $\mathrm{ml}$ 10-2M sodium sulphur.

c) id. a) + 0,6 $\mathrm{ml}$ 10-2M sodium sulphur.

\subsection{GROUPEMENTS SULFURÉS}

La réactivité de l'ion $\mathrm{Ag}^{+}$vis-à-vis des groupements sulfurés est très grande, et la précipitation des composés du type sulfure d'argent intervient dès le début de la courbe; pour les ultra-filtrats de lait 
les teneurs habituellement rencontrées n'ont pas d'influence significative sur la détermination des chlorures, mais pour des concentrations plus importantes, une interférence éventuelle est facilement détectée par une modification de la courbe avant le premier point équivalent; on pourrait éventuellement envisager d'effectuer leur détermination, en tenant compte des protons consommés, et d'évaluer ainsi l'ordre de grandeur de l'interférence provoquée (fig. 4).

\subsection{Acide lactique}

L'addition d'acide lactique ne modifie en rien l'allure des courbes de titrage. L'ion lactate ne réagit pas avec les ions $\mathrm{Ag}^{+}$à $\mathrm{pH}=8$.

\section{Applications aux ultrafiltrats de lait}

Nous avons comparé les résultats obtenus par notre méthode et ceux des méthodes spectrométrique et potentiométrique. La figure 5 montre les courbes de titrage obtenues pour deux ultra-

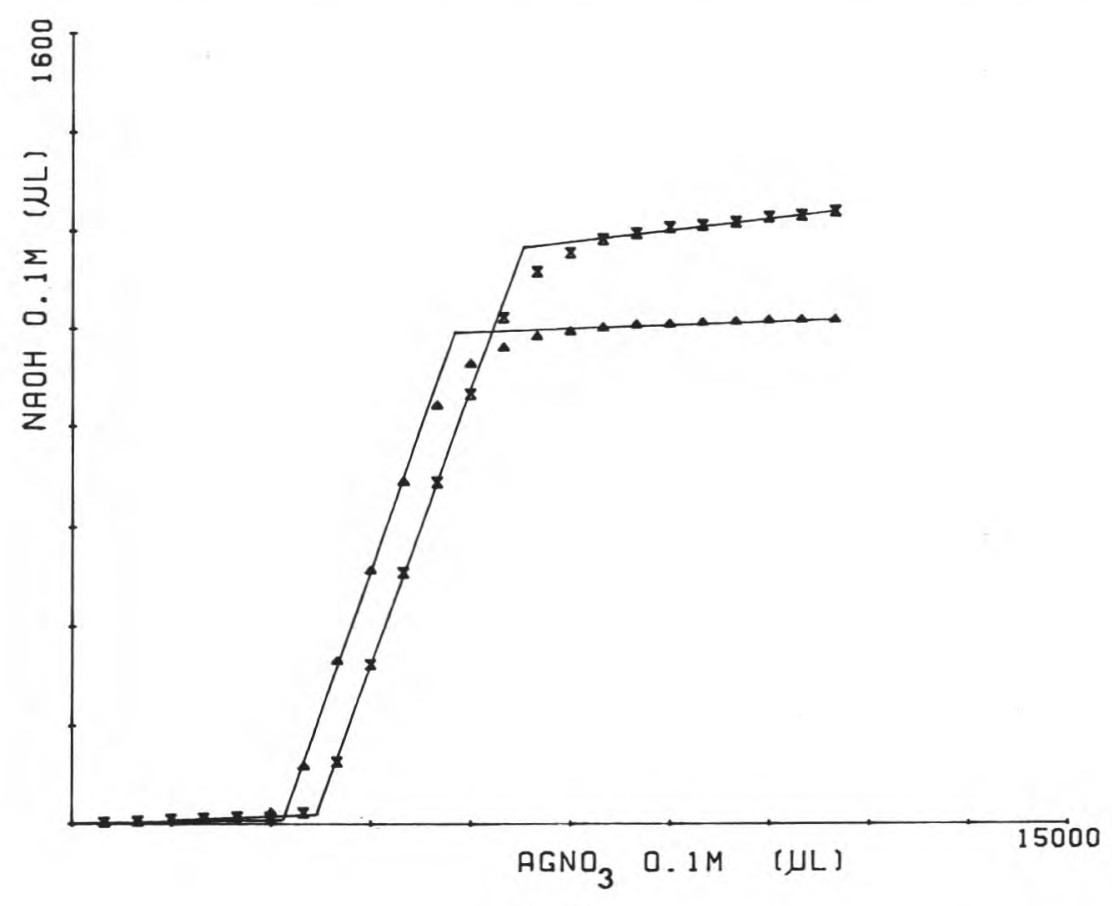

fig. 5

Courbes de titrage à $\mathrm{pH} 8$ obtenues pour des ultrafiltrats de lait $(10 \mathrm{ml}$ d'ultrafiltrats par du nitrate d'argent $0,1 \mathrm{M}$ ).

Titration curves at $\mathrm{pH} 8$ of milk ultrafiltrates $(10 \mathrm{ml}$ ultrafiltrates with $0,1 \mathrm{M}$ silver nitrate). 
(a)

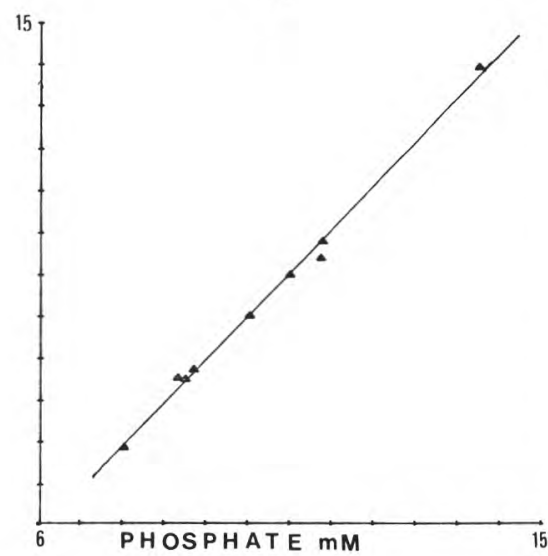

(b)

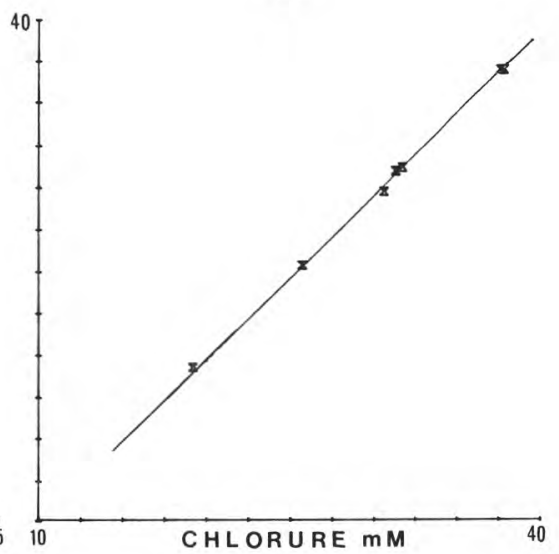

fig. 6

(a) Corrélation volumétrie-spectrométrie pour le dosage des phosphates dans des ultrafiltrats de lait.

(a) Correlation between volumetry and spectrometry for phosphate titration in milk ultrafiltrats.

(b) Corrélation volumétrie-potentiométrie pour le dosage des chlorures dans des ultrafiltrats de lait.

(b) Correlation between volumetry and potentiometry for chloride titration in milk ultrafiltrates.

filtrats. L'allure des courbes est identique à celles obtenues pour des milieux synthétiques.

La figure 6 montre la corrélation existant entre les différentes techniques. Chaque résultat est la moyenne de trois expériences. Pour le dosage des chlorures, on obtient un coefficient de corrélation de $r=0,9996$ et pour les phosphates $r=0,9975$. Nous avons testé la répétabilité de la méthode en faisant dix analyses d'un même ultrafiltrat; nous avons obtenu des coefficients de variation de 2,3\% pour les phosphates et 2,4\% pour les chlorures.

\section{CONCLUSION}

Le suivi d'un titrage argentimétrique des ions chlorure et phosphate réalisé à $\mathrm{pH}$ constant permet d'obtenir simultanément les teneurs de ces deux espèces. Le paramètre de mesure est le volume de soude nécessaire au maintien du $\mathrm{pH}$ constant; ce réactif est délivré par une burette automatique. La courbe de titrage obtenue 
présente trois segments de droite dont les intersections donnent les points équivalents. Le choix des conditions opératoires $(\mathrm{pH}=8$, déminéralisation préalable de l'échantillon, ajout d'un tampon borate) nous a permis d'éviter les interférences dues aux ions calcium, magnésium et aux acides aminés. Les résultats obtenus présentent une bonne cohérence avec ceux des méthodes classiques spectrométrique et potentiométrique.

Cette technique de titrage associée à la micro-informatique qui permet une exploitation rapide des courbes peut être appliquée au dosage de nombreuses espèces minérales importantes du lait. En effet, elle a précédemment été proposée pour le dosage des ions calcium et magnésium (Di Gregorio, Sisto 1980), des ions citrate (Le Nours, Le Meur, Bourgeois, 1982) et peut être envisagée pour d'autres espèces telles que groupements thiols.

\section{Bibliographie}

BRZENK (H. R.) and KRETT (O.J.) (1976). - Collaborative study of methods for the determination of chloride, citrate and phosphate in processed cheese. J. of the A.O.A.C., 59, 5, 1142-1145.

Christianson (G.), Jenness (R.) and Coulter (S. T.) (1954). - Determination of ionized calcium and magnesium in milk. Anal. Chem., 26 (12), 1923-1927.

Di GREgorio (F.) and Sisto (R.) (1980). - Use of o pH stat to indicate the end point in titration of calcium plus magnesium in milk and whey. J. Dairy Res., 47, 417-419.

Duval (L.) (1963). - Etude des conditions de validité du dosage céruléomolybdique de l'acide phosphorique. Chim. Anal., 45, 237-249.

LE Meur (J.F.) et Le Nours (H.) (1984). - Utilisation des données fournies par une chaîne de $\mathrm{pH}$ stat pour le dosage d'acides faibles. Modélisation des courbes. Analusis, 12, 36-41.

Le Nours (H.), Le Meur (J. F.) et Bourgeois (C.) (1982). - Dosage du citrate dans les ultrafiltrats de lait par titrage complexométrique avec le cuivre (II) à pH contrôlé. Sci, Aliments, 2, 483-493.

Sillen (L. G.) and MARTEll (A. E.) (1964). - Stability constants of metal in complexes. Special publication $\mathrm{n}^{\circ} 17$, the chemical Society, London.

Sleigh (R. W.), Sculley (T. B.) and Mackinlay (A. G.) (1979). - The binding of $\beta$-casein to hydroxyapatite: the effect of phosphate content and location. J. Dairy Res., 46, 337-342.

Visser (J.), Schaier (R. W.) and VAN GoRKom (M.) (1979). - The role of calcium, phosphate and citrate ions in the stabilization of casein micelles. $J$. Dairy Res., 46, 333-335.

White (J.C.D.) and DAvies (D. T.) (1958). - The relation between the chemical composition of milk and the stability of the caseinate complex. J. Dairy Res., 25, 236-295. 